\title{
Modelos factoriales de la Escala de Autoestima de Rosenberg en adolescentes peruanos
}

\author{
Andy Rick Sánchez-Villena, Valeria de La Fuente-Figuerola y José Ventura-León \\ Universidad Privada del Norte, Cajamarca, Perú
}

Factorial models of Rosenberg's Self-esteem Scale in Peruvian adolescents

\begin{abstract}
The Rosenberg Self-Esteem Scale (RSE) is an instrument widely used and designed to measure self-esteem in a single dimension. The aim of this research was to analyze the internal structure and the reliability of the RSE in Peruvian adolescents. The scale was administered to a total of 715 adolescents $(51.3 \%$ female) with an average age of 12.20 years. Six models were identified by confirmatory factor analysis, which correspond to factorial structures reported in previous studies. The results showed a better goodness of fit for the one-dimensional model with control of method effects on inverse items, eliminating item 8 due to its ambiguity. Reliability was adequate and was calculated using the omega coefficient. The unifactorial structure of the RSE is discussed and it is concluded that the instrument reports evidence of validity and adequate internal consistency to be used in the Peruvian context.
\end{abstract}

Keywords: Self-esteem; teenagers; validity; reliability.

Resumen: La Escala de Autoestima de Rosenberg (RSE) es un instrumento ampliamente utilizado que fue diseñado para medir la autoestima en una sola dimensión. La presente investigación tuvo como objetivo principal analizar la estructura interna y la fiabilidad de la RSE en adolescentes peruanos. La escala fue administrada a un total de 715 adolescentes $(51.3 \%$ de sexo femenino; $M$ edad = 12.20 años). Se identificaron seis modelos mediante análisis factorial confirmatorio, los cuales corresponden a estructuras factoriales reportadas en estudios previos. Los resultados mostraron mejores bondades de ajuste para el modelo unidimensional con control del efecto del método en ítems inversos eliminando el ítem 8 por su ambigüedad. La fiabilidad fue adecuada y se calculó a través del coeficiente omega. Se discute la estructura unifactorial de la RSE y se concluye que el instrumento proporciona evidencia de validez y consistencia interna adecuadas para ser utilizada en el contexto peruano.

Palabras clave: Autoestima; adolescentes; validez; fiabilidad.

\section{Introducción}

La autoestima es definida como un conjunto de pensamientos, sentimientos y creencias positivas o negativas respecto a uno mismo (Rosenberg, 1965, 1979; Ro-

Recibido: 03 de febrero 2020: aceptado: 26 de junio 2020

Correspondencia: Andy Rick Sánchez Villena, Universidad Privada del Norte, Av. Vía de Evitamiento s/n cdra 15, 06000 Cajamarca, Perú. Correo-e: andy.sanchez@upn.pe

Agradecimientos: Agradecemos a la Facultad de Psicología de la Universidad Privada Antonio Guillermo Urrelo. senberg et al., 1995). La autoestima también involucra autopercepciones sobre las propias habilidades, expectativas, relaciones sociales e inteligencia, expresando en qué medida uno se cree y siente exitoso o merecedor de algo (Souza y Cunba, 2019). Esta autoevaluación puede verse afectada por retroalimentación social a través de comentarios externos referidos al desempeño del individuo en sus diferentes roles (Gómez-Lugo et al., 2016) o también por una retroalimentación propia del individuo respecto a sus acciones (Luciano Gómez et al., 2002). Ante ello, la autoestima no es inalterable porque puede variar y cambiar en función de las diferentes 
etapas de la vida y de las experiencias cotidianas (Mayordomo et al., 2019).

La autoestima en los adolescentes está relacionada con variables académicas, personales, sociales y familiares. Así, por ejemplo, se ha encontrado relación de la autoestima con el bullying (Shahnaz y Ara, 2019), la satisfacción con la vida escolar (Park et al., 2016), el rendimiento académico (Orth, 2018) la soledad, la empatía, la depresión, la ansiedad, la salud mental en general (Moksnes y Reidunsdatter, 2019), la conducta suicida (Benavides-Mora et al., 2019), el apoyo social, la estabilidad y la satisfacción con las relaciones sociales (Park et al., 2016), el ambiente familiar, la calidad de la relación con los padres, el estatus socioeconómico de la familia y el castigo (Orth, 2018).

Para evaluar la autoestima existen múltiples instrumentos como la State Self Esteem Scale (SSES; Heatherton y Polivy, 1991), la Janis-Field Scale (Fleming y Courtney, 1984), la Colective Self Esteem Scale (CSES; Luhtanen y Crocker, 1992), el Social Self Esteem Inventory (SSEI; Lawson et al., 1979), la Multidimensional Scale of Self-Esteem (EMES-16; Barbot y Safont-Motay, 2019), el Inventario de Autoestima de Coopersmith (SEI; Coopersmith, 1967) y la Rosenberg Self-Esteem Scale (Rosenberg, 1965; versión española de Atienza et al., 2000), siendo esta última una de las más utilizadas a nivel mundial (cuenta con 3016 citas entre el periodo 2010-2014; Alessandri et al., 2015).

A pesar de su amplio uso, los estudios psicométricos han encontrado diferentes estructuras factoriales en la RSE (Goldsmith, 1986), lo cual es atribuido a la redacción de los ítems (Greenberger et al., 2003) y a las características culturales de las muestras (Schmitt y Allik, 2005). De este modo, se ha visto que la RSE en adolescentes muestra dos factores, los cuales denotan una autoevaluación positiva y negativa de sí mismo, por ello se los denomina autoestima positiva y autoestima negativa, pero ambos miden esencialmente un solo constructo cuando se incluye control del efecto en los ítems inversos (Quilty et al., 2006; Tomas y Oliver, 1999). Este último modelo es invariante según el sexo (Tomas et al., 2015). Otros estudios señalan que la RSE es unidimensional cuando todos los ítems están redactados únicamente de forma positiva o negativa, pero es bidimensional cuando se tiene en cuenta la versión original con cinco items inversos (Greenberger et al., 2003). Por otro lado, se ha reportado que el modelo bifactor de la RSE fue el más satisfatorio, mostrando mejores bondades de ajuste (Alessandri et al., 2015; Gnambs et al, 2017). No obstante, se sabe que el modelo bifactor de la RSE podría mostrar dichos resultados porque estos se acomodan mejor a modelos implausi- bles o a patrones inválidos de respuesta, por lo que la RSE podría ser esencialmente unidimensional (Reise et al., 2016). Otro estudio llevado a cabo con adolescentes latinos indicó que el modelo de dos factores correlacionados, teniendo en cuenta los efectos del método, mostraba mejor ajuste frente al modelo unidimensional de la RSE (Supple y Plunkett, 2011). Sin embargo, en un estudio posterior los mismos autores señalaron que «es dificil concluir definitivamente que la bidimensionalidad está relacionada con los efectos del método» (Supple et al., 2013, p. 761).

En el contexto latinoamericano, Cogollo et al. (2015) realizaron un análisis factorial exploratorio con adolescentes de Colombia, cuyos resultados mostraron que la RSE era un constructo bidimensional. En Brasil, una investigación encontró que este instrumento se ajustaba mejor como constructo unidimensional (Santos y Maia, 2003), mientras que el estudio de Burges et al. (2010) encontró mejor ajuste en el modelo de dos factores. En Argentina, se encontró que la RSE era un constructo unidimensional (Góngora et al., 2010). Por último, en Perú se encontró que el modelo bifactor junto al modelo bidimensional tuvieron mejores índices de bondad de ajuste. Sin embargo, después de usar índices de resistencia como la varianza común explicada (ECV), el porcentaje de correlaciones no contaminadas (PUC) y el coeficiente omega jerárquico optaron por el segundo (Ventura-León et al., 2018).

Por lo expuesto, el objetivo principal de la presente investigación es analizar las propiedades psicométricas de la RSE a través de la evaluación de seis modelos factoriales y el cálculo de la fiabilidad de las puntuaciones en adolescentes peruanos. Como objetivos específicos se plantean: a) comparar dichos modelos y b) seleccionar el que presente mejor bondad de ajuste. En tal sentido, el propósito de la investigación es brindar evidencias de validez y fiabilidad que respalden el uso de este instrumento. Esto podría posibilitar que los profesionales de la salud mental puedan lograr mayor precisión en la evaluación de la autoestima en adolescentes, la cual es parte importante de su bienestar psicológico (Das et al., 2016; Moksnes y Reidunsdatter, 2019).

\section{Método}

\section{Participantes}

En este estudio participaron 715 adolescentes estudiantes $(51.3 \%$ mujeres) de instituciones públicas $(50.3 \%)$ y privadas $(49.7 \%)$ de la ciudad de Cajamarca, Perú, cuyas edades oscilan entre los 10 y los 17 años ( $M$ $=12.20 ; D T=1.89$ ). 


\section{Procedimiento}

Para la realización del presente estudio se siguieron las recomendaciones del International Test Commission (ITC, 2017). Inicialmente, se solicitó permiso a las autoridades académicas de las respectivas instituciones educativas. Se coordinó con los departamentos de psicología y se procedió con la administración del instrumento a los estudiantes. Durante dicha sesión de administración se indicó a los participantes que no eran necesarios sus datos personales ni nada que los puediera identificar, ya que su participación era totalmente voluntaria y anónima. Es importante mencionar que los padres de los estudiantes fueron informados del estudio y firmaron los consentimientos respectivos.

\section{Instrumentos}

Escala de autoestima de Rosenberg (RSE; Rosenberg, 1965; versión en español de Atienza et al., 2000). La RSE es una escala de autoinforme que evalúa la autoestima y consta de 10 ítems (5 inversos) con cuatro opciones de respuesta tipo Likert que van desde muy en desacuerdo $=1$ hasta muy de acuerdo $=4$. Por lo tanto, tiene un rango de puntuaciones totales de 10 a 40. La escala es unidimensional tanto en la versión original como en la versión en español. La validez y fiabilidad de la versión traducida se realizó mediante análisis factorial confirmatorio mostrando un mejor ajuste en el modelo unidimensional. La fiabilidad se obtuvo mediante el coeficiente alfa de Cronbach $(\alpha=.86)$. Además, se encontró estabilidad temporal con el método de test-retest, cuyos valores oscilaron entre $r=.85$ y $r=.88$. En Perú, este instrumento ha sido validado por Ventura-León et al. (2018) mostrando adecuadas propiedades psicométricas para el modelo bidimensional: autoestima positiva $(\omega=.803)$ y autoestima negativa $(\omega=.723)$.

\section{Análisis estadístico}

Primero, se hizo un análisis preliminar de los ítems con el software estadístico Jamovi teniendo como punto de corte valores de curtosis y asimetría que no superaran el \pm 1.5 para así determinar proximidad a la normalidad univariada (Pérez y Medrano, 2010). Con el objetivo de examinar normalidad multivariada se calculó el coeficiente de Mardia, esperando valores inferiores a 70. Posteriormente, se realizó un análisis factorial confirmatorio (AFC) con el software AMOS versión 22. Como método de estimación se empleó máxima verosimilitud (ML) debido a la normalidad de los datos. Para determinar el ajuste del modelo se tuvieron en cuenta diferentes índices de bondad de ajuste junto a los puntos de corte propuestos por $\mathrm{Hu}$ y Bentler (1999), como el índice de ajuste comparativo $(\mathrm{CFI} \geq .95)$, la raíz cuadrática media del error estandarizado (SRMR $\leq .08)$ y la raíz media del error estandarizado (RMSEA < .06). Para determinar la comparación entre los diferentes modelos se tuvo en cuenta la razón de $\chi^{2} / g l$ donde valores inferiores a 2 son adecuados (Tabachnick y Fidell, 2007) y el criterio de información bayesiano (BIC), cuyos valores inferiores indican un modelo con mejor ajuste frente a los otros (Góngora, 2011).

A continuación se probaron seis modelos diferentes (ver la Figura 1): el primero con una estructura unidimensional con los 10 ítems originales; el segundo con una estructura unidimensional retirando el ítem 8 (Ventura-León et al., 2018) debido a su ambigüedad (Schmitt y Allik, 2005); el tercero con una estructura bidimensional correspondiente a los factores autoestima positiva y autoestima negativa, los cuales se encuentran correlacionados (modelo oblícuo); el cuarto con una estructura bidimensional correspondiente a los factores autoestima positiva y autoestima negativa sin correlación (modelo ortogonal); el quinto con una estructura unidimensional con control del efecto del método en los ítems inversos; el sexto fue un modelo bifactor, donde se consideraron las dos dimensiones correspondientes a autoestima positiva y negativa, con un factor general. Cabe señalar que este último se dejó de lado debido al error de varianza negativa, el cual indica que el modelo puede ser incorrecto (Joreskog y Sorbom, 1984). Dicha situación también ha sido reportada en estudios previos con población latina (Supple et al., 2013). Finalmente, la fiabilidad se calculó mediante el coeficiente omega (Ventura-León y Caycho-Rodríguez, 2017), considerados adecuados los valores $\geq .70$.

\section{Resultados}

\section{Análisis preliminar de los items}

El análisis inicial de los datos se muestra en la Tabla 1 , en donde se observa que la curtosis y asimetría indican normalidad univariada, dado que no superan el valor de \pm 1.5 (Pérez y Medrano, 2010). El coeficiente de mardia fue de 23.53, indicando normalidad multivariada. Ante ello se optó por utilizar $M L$ como método de estimación en el AFC. 


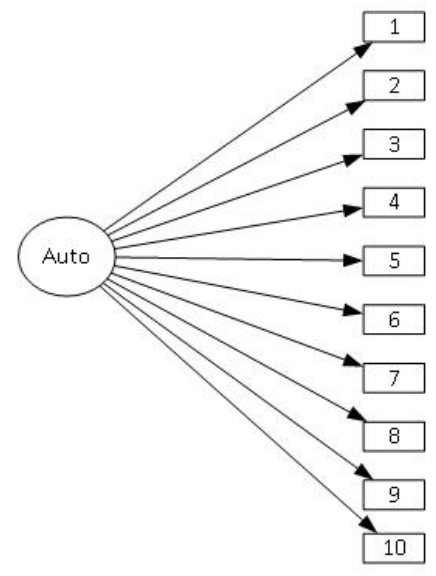

Modelo 1
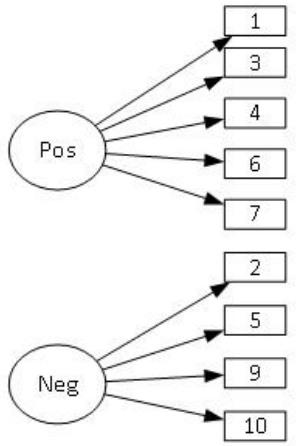

Modelo 4

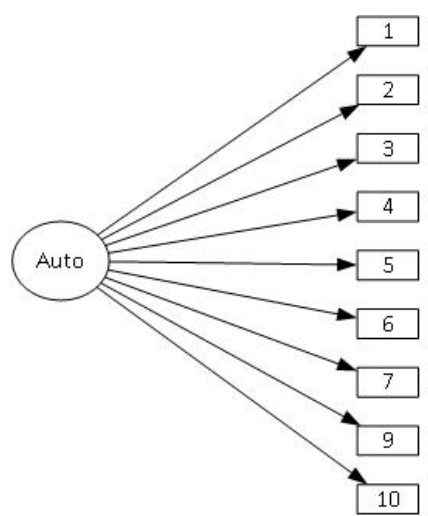

Modelo 2

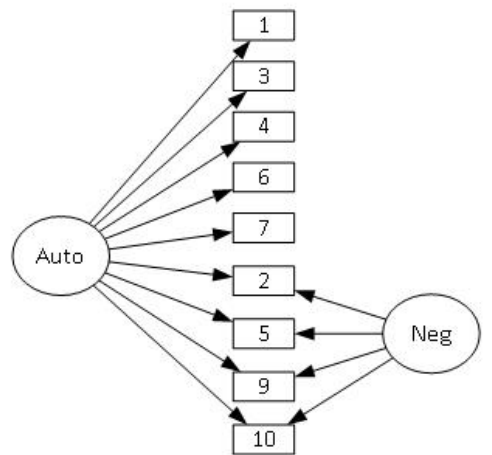

Modelo 5

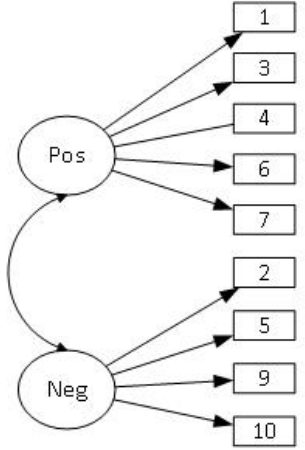

Modelo 3

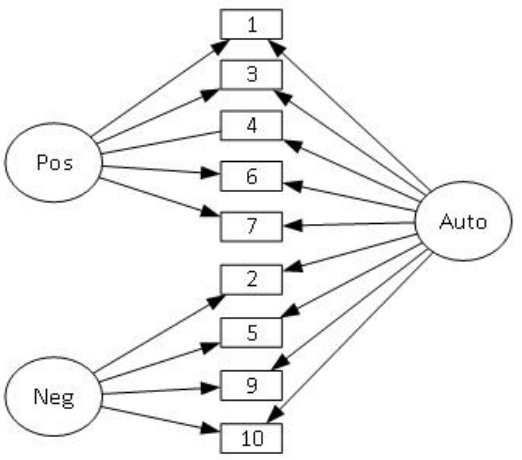

Modelo 6

Figura 1. Modelos Factoriales de la RSE.

Tabla 1. Estadísticos descriptivos de la RSE

\begin{tabular}{ccccc}
\hline Ítems & $M$ & $D T$ & Asimetría & Curtosis \\
\hline Ítem 1 & 3.35 & 0.81 & -1.22 & 1.02 \\
Ítem 2* & 3.13 & 0.88 & -0.79 & -0.13 \\
Ítem 3 & 3.37 & 0.72 & -1.20 & 1.68 \\
Ítem 4 & 3.22 & 0.85 & -0.93 & 0.22 \\
Ítem 5* & 2.71 & 1.05 & -0.28 & -1.12 \\
Ítem 6 & 3.31 & 0.83 & -1.12 & 0.63 \\
Ítem 7 & 3.32 & 0.82 & -1.11 & 0.65 \\
Ítem 8* & 1.71 & 0.83 & 1.14 & 0.89 \\
Ítem 9* & 2.81 & 1.02 & -0.25 & -1.15 \\
Ítem 10* & 3.07 & 1.01 & -0.68 & -0.77 \\
\hline
\end{tabular}

Nota. $M=$ media aritmética; $D T=$ desviación típica. *ítems inversos

\section{Análisis factorial confirmatorio}

Los resultados del AFC se muestran en la Tabla 2, donde se observa que dos modelos obtuvieron las mejores bondades de ajuste: el modelo bidimensional con factores correlacionados (modelo 3 ) y el modelo unidi- 
mensional con control del efecto en los ítems inversos. Ambos obtuvieron índices similares con CFI > .95, RM$\mathrm{SEA}<.06$ y $\mathrm{SRMR}<.08$. Teniendo en cuenta el valor más bajo de BIC y la razón de $\chi^{2} / g l$, seleccionaríamos al modelo 3 como el mejor; no obstante dicha decisión se discutirá en la sección correspondiente.

Tabla 2. Modelos factoriales de la RSE

\begin{tabular}{|c|c|c|c|c|c|c|c|}
\hline Modelos & $\chi^{2}$ & $\mathrm{gl}$ & $\chi^{2} / \mathrm{gl}$ & CFI & $\begin{array}{c}\text { RMSEA } \\
\text { [IC90\%] }\end{array}$ & $S R M R$ & $B I C$ \\
\hline Modelo 1 & 236.42 & 35 & 6.76 & .808 & $\begin{array}{c}.090 \\
{[.079 ; .101]}\end{array}$ & .064 & 367.87 \\
\hline Modelo 2 & 214.55 & 27 & 7.94 & .818 & $\begin{array}{c}.099 \\
{[.087 ; .111]}\end{array}$ & .066 & 332.76 \\
\hline Modelo 3 & 63.83 & 26 & 2.46 & .963 & $\begin{array}{c}.045 \\
{[.031 ; .059]}\end{array}$ & .035 & 188.70 \\
\hline Modelo 4 & 200.47 & 27 & 7.43 & .832 & $\begin{array}{c}.095 \\
{[.083 ; .107]}\end{array}$ & .132 & 318.77 \\
\hline Modelo 5 & 59.08 & 23 & 2.57 & .965 & $\begin{array}{c}.047 \\
{[.032 ; .062]}\end{array}$ & .033 & 203.67 \\
\hline
\end{tabular}

Nota . BIC = criterio de información bayesiano; CFI = índice de ajuste comparativo; RMSEA = raiz cuadrática media del error de aproximación; $S R M R$ = raíz media del error estandarizado; La negrita indica los modelos con mejor ajuste.

Las cargas factoriales de los diferentes modelos, junto a la fiabilidad calculada, se muestran en la Tabla 3. Tras analizar las saturaciones se encontró que todas superan el .30 (Kline, 2015) a excepción del ítem 8 del modelo 1, el cual también es inverso. En consecuencia, este ítem se excluyó de los siguientes análisis. Asimis- mo, el ítem 5 mostró cargas por debajo de .30 en los siguientes modelos. Finalmente, la fiabilidad fue adecuada al superar el .70 en todos los modelos a excepción del modelo 1 y 5 , los cuales, si bien no superan el punto de corte, sí se hallan muy próximos.

Tabla 3. Cargas factoriales de la RSE

\begin{tabular}{|c|c|c|c|c|c|}
\hline Item & Modelo 1 & Modelo 2 & Modelo 3 & Modelo 4 & Modelo 5 \\
\hline 1 & .500 & .495 & .568 & .566 & .569 \\
\hline 2 & .586 & .590 & .625 & .600 & $.424 / .438$ \\
\hline 3 & .361 & .355 & .448 & .482 & .451 \\
\hline 4 & .471 & .468 & .543 & .548 & .541 \\
\hline 5 & .190 & .195 & .211 & .210 & $.121 / .171$ \\
\hline 6 & .516 & .512 & .571 & .556 & .573 \\
\hline 7 & .510 & .503 & .567 & .554 & .564 \\
\hline 8 & -.113 & - & - & - & - \\
\hline 9 & .537 & .543 & .618 & .617 & $.348 / .509$ \\
\hline 10 & .641 & .648 & .771 & .794 & $.441 / .662$ \\
\hline$\omega$ & .694 & .732 & .798 & .799 & $.698 / .507$ \\
\hline
\end{tabular}

Nota. Las cargas en negrita pertenecen al control del método.

\section{Discusión}

La autoestima, además de ser una variable ampliamente estudiada, es un aspecto que incide en la salud mental de la población adolescente (Das et al., 2016; Moksnes y
Reidunsdatter, 2019). En consecuencia, es importante realizar estudios psicométricos que permitan respaldar con evidencias de validez y fiabilidad el uso de la RSE (Rosenberg, 1965) como herramienta para la medición y evaluación de la autoestima en adolescentes por parte de 
los profesionales de la salud mental. Por ello, el objetivo principal de esta investigación fue analizar las propiedades psicométricas de dicha escala teniendo en cuenta que es una de las más utilizadas a nivel mundial (Alessandri et al., 2015). Como objetivos específicos planteamos a) comparar los diferentes modelos factoriales propuestos por estudios anteriores y b) seleccionar el mejor modelo a fin de ser utilizado en el contexto peruano.

En relación al objetivo general, a través del AFC se modelaron seis estructuras factoriales de la RSE encontradas en la literatura científica (Goldsmith, 1986; Quilty et al., 2006; Schmitt y Allik, 2005; Tomas y Oliver, 1999; Ventura-León et al., 2018). En primer lugar, los resultados mostraron que el modelo 6 , correspondiente al modelo bifactor, fue omitido debido al error de varianza negativa (Joreskog y Sorbom, 1984). Posteriormente se encontró que los modelos 3 y 5 , correspondientes al modelo bidimensional oblícuo y al unidimensional con control del efecto, respectivamente, obtuvieron los mejores índices de bondad de ajuste. En el caso del modelo 3, los hallazgos coinciden con los antecedentes internacionales (Burges et al., 2010; Chiu, 1988, Cogollo et al., 2015; Goldsmith, 1986; Supple y Plunkett, 2011) y nacionales (Ventura-León et al., 2018); no obstante, la dimensión de autoestima negativa puede deberse a la redacción de los ítems, ya que los reactivos son inversos (Greenberger et al., 2003) y, en consecuencia, la interpretación de ambas dimensiones no estaría justificada. Respecto al modelo 5, los resultados son coherentes con estudios internacionales (Quilty et al., 2006; Schmitt y Allik, 2005), principalmente con los de Tomas y Oliver (1999) y Tomas et al. (2015), quienes encontraron que la RSE es unidimensional cuando se controla el efecto del método en los ítems inversos. Consideramos que esta estructura es más plausible porque: a) la correlación entre factores es de .59 , un valor nada despreciable que sugeriría unidimensionalidad (Reise, 2012); b) si bien los modelos bifactor de la RSE han demostrado resultados óptimos (Alessandri et al., 2015; Gnambs et al., 2017), estos tienden a ajustar mejor los datos en situaciones poco verosímiles por lo que el modelo bifactor podría indicar escencialmente unidimensionalidad en la RSE; c) los índices de bondad de ajuste encontrados en el modelo 3 y 5 tienen diferencias mínimas y, en consecuencia, optar por la estructura unifactorial de la RSE sería más prudente por ser más parsimonioso; d) decidir a favor del modelo de un solo factor sería coherente con la teoría que subyace a la construcción de la RSE y a la conceptualización de la autoestima global (Rosenberg, 1965, 1979; Rosenberg et al., 1995). Como resultado, considerar a la RSE como unidimensional en la población de adolescentes peruanos, coincidiría con investigaciones hechas a nivel de
Latinoamérica (Burges et al., 2010; Góngora et al., 2010; Santos y Maia, 2003; Supple y Plunkett, 2011; Supple et al., 2013). Por lo tanto, al comparar ambos modelos, optamos por el modelo 5, cumpliendo con el objetivo específico de seleccionar el modelo con mejor ajuste.

Cabe señalar que se retiró el ítem 8 por dos razones: por recomendación del antecedente nacional (Ventura-León et al., 2018) y porque a nivel de Latinoamérica, Asia y África se ha reportado que es un ítem ambiguo (Schmitt y Allik, 2005) con carga factorial inversa, lo cual podría deberse a una mala interpretación del mismo, siendo, por el contrario, un indicador de alta autoestima pues denota un deseo de mayor respeto (si yo me estimo, entonces merezco más respeto) o que ya posee un nivel adecuado de respeto hacia sí mismo. Respecto a la fiabilidad, el coeficiente omega del modelo 5 mostró un valor de .698 , siendo muy próximo al .70 sugerido, lo cual indica una adecuada consistencia interna de la RSE unidimensional con control del efecto del método.

En conclusión, los resultados muestran que la RSE en población adolescente peruana debería ser interpretada como unidimensional debido a las adecuadas bondades de ajuste, fiabilidad y a la parsimonia, ademaás de a la coherencia que tendría frente a la teoría subyacente (Rosenberg, 1965, 1979; Rosenberg et al., 1995).

Respecto a las limitaciones del estudio, se destaca que los participantes fueron seleccionados de manera no probabilística, lo que afectaría a la extrapolación de los resultados; sin embargo, esto se trató de mitigar seleccionando una cantidad considerable de adolescentes, siendo considerado $n>500$ individuos como una muestra adecuada para estudios psicométricos (Kline, 2015). Una segunda limitación es que la muestra fue tomada en una región específica de Perú. En este sentido, se sugiere que proximas investigaciones repliquen el estudio con participantes seleccionados al azar y en diferentes regiones del país para poder lograr una mayor generalizabilidad de los resultados. También se sugiere un mayor control del ítem 5 por su baja carga factorial. Finalmente, se requiere que futuros estudios puedan demotrar la validez convergente y divergente de la RSE para evidenciar la validez basada en la relación con otras variables. Pese a estas limitaciones, la presente investigación proporciona información valiosa sobre la utilidad y la estructura factorial de la RSE en adolescentes peruanos.

\section{Referencias}

Alessandri, G., Vecchione, M., \& Eisenberg, N. (2015). On the factor structure of the Rosenberg (1965) General Self-Esteem Scale. Psychological Assessment, 27, 621-635. https://doi. org/10.1037/pas0000073 
Atienza, F. L., Moreno, Y. y Balaguer, I. (2000). Análisis de la dimensionalidad de la Escala de Autoestima de Rosenberg en una muestra de adolescentes valencianos. Revista de Psicología. Universitas Tarraconensis, 23, 29-42.

Barbot, B., \& Safont-Motay, C. (2019). Multidimensional Scale of Self-esteem (EMES-16): psychometric evaluation of a domain-specific measure of self-esteem for French-speaking adolescents. International Journal of Behavioral Development, 43, 1-11. https://doi.org/10.1177/0165025418824996

Benavides-Mora, V. K., Villota-Melo, N. G. y Villalobos-Galvis, F. H. (2019). Conducta suicida en Colombia: una revisión sistemática. Revista de Psicopatología y Psicología Clínica, 24, 181-195. https://doi.org/10.5944/rppc.24251

Burges, J., Ruschel, D. y Daibosco, D. (2010). Escala de Autoestima de Rosenberg (RSE): validade fatorial e consistência interna. Psico-USF, 15, 395-403. https://doi. org/10.1590/S1413-82712010000300012

Cogollo, Z., Campo-Arias, A. y Herazo, E. (2015). Escala de Rosenberg para autoestima: consistencia interna y dimensionalidad en estudiantes de Cartagena, Colombia. Psicología: Avances de la Disciplina, 9, 61-71. https://doi. org/10.21500/19002386.1814

Coopersmith, S. (1967). The antecedents of self-esteem. W.H. Freeman \& Co.

Das, J. K., Salam, R., Lassi, Z. S., Khan, M. N., W, M., Patel, V., $\&$ Bhutta, Z. A. (2016). Interventions for adolescent mental health: an overview of systematic reviews. Journal of Adolescent Health, 59, 49-60. https://doi.org/10.1016/j. jadohealth.2016.06.020

Fleming, J., \& Courtney, B. (1984). The dimensionality of selfesteem: II. Hierarchical facet model for revised measurement scales. Journal of Personality and Social Psychology, 46, 404421. https://doi.org/10.1037/0022-3514.46.2.404

Gnambs, T., Scharl, A., \& Schroeders, U. (2017). The structure of the Rosenberg Self-Esteem Scale: A cross cultural metaanalysis. Zeitschrift für Psychologie, 226, 14-29. https://doi. org/10.1027/2151-2604/a000317

Goldsmith, R. E. (1986). Dimensionality of the Rosenberg SelfEsteem Scale. Journal of Social Behavior and Personality, 1, 253-264.

Gómez-Lugo, M., Espada, J., Morales, A., Marchal-Bertrand, L., Soler, F. y Vallejo-Medina, P. (2016). Adaptation, validation, reliability and factorial equivalence of the Rosenberg SelfEsteem Scale in Colombian and Spanish population. The Spanish Journal of Psychology, 19, 1-12. https://doi. org/10.1017/sjp.2016.67

BIBLIOGRAPHY Góngora, R. (2011). Prueba de hipótesis anidadas y no anidadas y métodos de discriminación para modelos no lineales. (Tesis de Maestría), Centro de Investigación en Matemáticas, A.C., Guanajuato, México.

Góngora, V., Fernández, M. y Castro, A. (2010). Estudio de validación de la Escala de Autoestima de Rosenberg en población adolescente de la ciudad de Buenos Aires. Perspectivas en Psicología, 7, 24-30.

Greenberger, E., Chen, C., Dmitrieva, J., \& Farruggia, S. P. (2003). Item-wording and the dimensionality of the Rosenberg Selfesteem Scale: do they matter? Personality and Individual Differences, 35, 1241-1254. https://doi.org/10.1016/S01918869(02)00331-8
Heatherton, T., \& Polivy, J. (1991). Development and validation of a scale for measuring state self-esteem. Journal of Personality and Social Psychology, 80, 895-910. https://doi.org/10.1037/00223514.60.6.895

Hu, L. T., \& Bentler, P. M. (1999). Cutoff criteria for fit indexes in covariance structure analysis: conventional criteria versus new alternatives. Structural Equation Modeling, 6, 1-55. https://doi.org/10.1080/10705519909540118

International Test Commission. (2017). The ITC guidelines for translating and adapting tests. Version, 1.0.

Joreskog, K.G. \& Sorbom, D. (1984). LISREL-VI user's guide (3rd ed.). Scientific Software.

Kline, P. (2015). A handbook of test construction (psychology revivals): Introduction to psychometric design. Routledge.

Lawson, J. S., Marshall, W. L., \& McGrath, P. (1979). The Social SelfEsteem Inventory. Educational and Psychological Measurement, 39, 803-811. https://doi.org/10.1177/001316447903900413

Luciano M. C., Gómez I. y Valdivia S. (2002). Consideraciones acerca del desarrollo de la personalidad desde un marco funcional-contextual. International Journal of Psychology and Psychological Therapy, 2, 173-197.

Luhtanen, R., \& Crocker, J. (1992). A Colective Self-Esteem Scale: self-evaluation of one's social identity. Personality and Social Psychology Bulletin, 18, 302-318. https://doi. org/10.1177/0146167292183006

Mayordomo, T., Gutierrez, M., \& Sales, A. (2019.). Adapting and validating the Rosenberg Self-Esteem Scale for elderly Spanish population. International Psychogeriatrics, 32, 183-190. https://doi.org/10.1017/S1041610219001170

Moksnes, U. K., \& Reidunsdatter, J. (2019). Self-esteem and mental health in adolescents-level and stability during a school year. Norks Epidemiologi, 28, 59-67. https://doi.org/10.5324/nje. v28i1-2.3052

Orth, U. (2018). The family environment in early childhood has a long-term effect on self-esteem: A longitudinal study from birth to age 27 years. Journal of personality and social psychology, 114, 637-655. https://doi.org/10.1037/pspp0000143

Park, J., Kim, Park, S. J., Suh, S., \& Lee, H. J. (2016). The relationship between self-esteem and overall health behaviors in Korean adolescents. Heal Psychology and Behavioral Medicine, 4, 175-185. https://doi.org/10.1080/21642850.2016.1246971

Pérez, E. R. y Medrano, L. A. (2010). Análisis factorial exploratorio: bases conceptuales y metodológicas. Revista Argentina de Ciencias del Comportamiento, 2, 58-66.

Quilty, L. C., Oakman, J., \& Risko, E. (2006). Correlates of the Rosenberg Self-Esteem Scale method effects. Structural Equation Modeling: A Multidisciplinary Journal, 13, 99-117. https://doi.org/10.1207/s15328007sem1301_5

Reise, S. P. (2012). The rediscovery of bifactor measurement models. Multivariate Behavioral Research, 47, 667-696. https://doi.org/10.1080/00273171.2012.715555

Reise, S. P., Kim, D. S., Mansolf, M., \& Widaman, K. F. (2016). Is the bifactor model a better model os is it just better at modeling implausible responses? application of interatively reweighted least squares to the Rosenberg Self-Esteem Scale. Multivariate Behavioral Research, 51, 818-838. https://doi.org/10.1080/00 273171.2016.1243461

Rosenberg, M. (1965). Society and the adolescent self image. Princeton University Press. 
Rosenberg, M. (1979). Conceiving the Self. Basic Books.

Rosenberg, M., Schooler, C., Schienbach, C. \& Rosenberg, F. (1995). Global self-esteem and specific self-Steem: different concepts, different outcomes. American Sociological Review, 60, 141-156. https://doi.org/10.2307/2096350

Santos, P. J., y Maia, J. (2003). Análise factorial confirmatória e validação preliminar de uma versão portuguesa da escala de auto-estima de Rosenberg. Psicologia: teoria, investigaçäo e prática, 2, 253-268.

Schmitt, D., \& Allik, J. (2005). Simultaneous administration of the Rosenberg Self-Esteem Scale in 53 nations: exploring the universal and culture-specific features of global self-esteem. Journal of Personality and Social Psychology, 89, 623-642. https://doi.org/10.1037/0022-3514.89.4.623

Shahnaz, I., \& Ara, H. (2019). Self-esteem and coping skills of victims and non-victims of bullying in public school. Dhaka University Journal of Biological Sciences, 28, 139-146.

Souza, T.J. \& Cunba, L.E. (2019). Rosenberg Self-Esteem Scale: method effect and gender invariance. Psico-USF, 24, 517528. https://doi.org/10.1590/1413-82712019240309

Supple, A. J., \& Plunkett, S. W. (2011). Dimensionality and validity of the Rosenberg Self-Esteem Scale for use with Latino adolescents. Hispanic Journal of Behavioral Sciences, 33, 39-53. https://doi.org/10.1177/0739986310387275
Supple, A. J., Su, J., Plunkett, S. W., Peterson, G. W., \& Bush, K. R. (2013). Factor structure of the Rosenberg Self-Esteem Scale. Journal of Cross-Cultural Psychology, 44, 748-764. https://doi.org/10.1177/0022022112468942

Tabachnick, B. G., \& Fidell, L. S. (2007). Using multivariate statistics (5th ed.). Pearson/Allyn \& Bacon.

Tomas, J. M., \& Oliver, A. (1999). Rosenber's self-esteem scale: two factors of method effects. Structural Equation Modeling: A multidisciplinary Journal, 6, 84-98. https://doi. org/10.1080/10705519909540120

Tomas, J. M., Oliver, A., Hontangas, P. M., Sancho, P., \& Galiana, L. (2015). Method effects and gender invariance of the Rosenberg Self-esteem Scale: A study on adolescents. Acta de Investigación Psicológica, 5, 2194-2203. https://doi. org/10.1016/S2007-4719(16)30009-6

Ventura-León, J. L. y Caycho-Rodríguez, T. (2017). El coeficiente Omega: un método alternativo para la estimación de la confiabilidad. Revista Latinoamericana de Ciencias Sociales, Niñez y Juventud, 15, 625-627.

Ventura-León, J., Caycho-Rodriguez, T., Barboza-Palomino, M. y Salas, G. (2018). Evidencias psicométricas de la Escala de Autoestima de Rosenberg en adolescentes limeños. Revista Interamericana de Psicología, 52, 44-60. 
Anexo 1.

Escala de Autoestima de Rosenberg (RSE)

Instrucciones: Por favor, lee las frases que figuran a continuación y señala el nivel de acuerdo o desacuerdo que tienes con cada una de ellas, marcando con un aspa la alternativa elegida.

\begin{tabular}{|c|c|c|c|}
\hline 1 & 2 & 3 & 4 \\
\hline Muy en desacuerdo & En desacuerdo & De acuerdo & Muy de acuerdo \\
\hline
\end{tabular}

\begin{tabular}{|l|l|l|l|l|}
\cline { 2 - 3 } \multicolumn{1}{l|}{} & \multicolumn{1}{c|}{1} & 2 & 3 & 4 \\
\hline 1. Me siento una persona tan valiosa como las otras & & & & \\
\hline 2. Casi siempre pienso que soy un fracaso* & & & & \\
\hline 3. Creo que tengo algunas cualidades buenas & & & & \\
\hline 4. Soy capaz de hacer las cosas tan bien como los demás & & & & \\
\hline 5. Pienso que no tengo mucho de lo que estar orgulloso* & & & & \\
\hline 6. Tengo una actitud positiva hacia mí mismo & & & & \\
\hline 7. Casi siempre me siento bien conmigo mismo & & & & \\
\hline 8. Me gustaría tener más respeto por mí mismo* (omitido) & & & & \\
\hline 9. Realmente me siento inútil en algunas ocasiones* & & & & \\
\hline 10. A veces pienso que no sirvo para nada* & & & & \\
\hline
\end{tabular}

*ítems inversos

Clave de corrección: Se invierten las puntuaciones de los items inversos (Muy en desacuerdo $=4$, En desacuerdo $=3$, De acuerdo $=2$, Muy de acuerdo = 1) y se suman las puntuaciones de los 9 ítems para obteneruna puntuación total (rango: 9-36). 
